\title{
La contribución de las energías renovables en la planificación energética española
}

\author{
Blanca Azcárate y Alfredo Mingorance
}

\section{LOS PRIMEROS PLANES ENERGÉTICOS}

Con la apertura al exterior de la economía española a partir de la entrada en vigor del Plan de Estabilización de 1959, el sector energético se vió profundamente afectado al poder acceder a un petróleo más barato en los mercados internacionales, lo que significó su utilización de manera intensiva en el proceso de desarrollo industrial que tuvo lugar en España durante la década de los sesenta.

Este desarrollo industrial llevó consigo un aumento de la demanda de energía entre $1963 / 73$ en un $128 \%$ (de 36,9 millones de Tec a 84 millones de $\mathrm{Tec}$ ). Por primera vez el petróleo desplaza al carbón como principal fuente de energía en el balance energético nacional (IRANZO, 1992).

A raiz de la crisis de 1973, originada por la guerra árabe israeli, surge una espectacular escalada de los precios del petróleo. Este acontecimiento se traduce en la necesidad de elaborar una planificación energética global ante la inseguridad del autoabastecimiento energético, para minimizar futuros riesgos.

Se elabora, entonces, el I Plan Energético Nacional (PEN'75), cuyo objetivo fundamental era la reducción del petróleo dentro del balance energético nacional, fomentando el carbón nacional, la hidroelectricidad y la energía termonuclear, para conseguir una mayor autosuficiencia energética.

Este primer plan desaparece con el cambio político, y se configura el segundo plan, el PEN 1977, con una vigencia prevista de diez años, que plantea una disminución de la dependencia del petróleo más realista que la establecida en el PEN anterior (los objetivos de la demanda del petróleo 
en el PEN'75 era del $43,7 \%$, en el PEN'77 es del $53,8 \%$ ). Este plan nunca entró en vigor, pero sirvió de base para la elaboración del Plan Energético Nacional 1978 (PEN'78), con el que se trataba de hacer frente a las costosas repercusiones que para la balanza de pagos suponian las importaciones energéticas, centradas en exceso en el crudo petrolífero. Este deseo de potenciar el autoabastecimiento energético determinó un programa acelerado de construcción de centrales térmicas de carbón, y el apoyo de la energia nuclear, lo que favorecía claramente la explotación de las cuencas carboníferas españolas, y por tanto, la energía fósil.

En definitiva, estos primeros planes energéticos, y sus posteriores revisiones, centraban el consumo energético exclusivamente en los combustibles fósiles, con una creciente participación de la energía nuclear (primera y segunda generación de centrales).

La planificación energética de la etapa socialista coincide con un nuevo plan, el PEN 1983-92, que tiene que afrontar todavía, una escasa producción de energía nacional y una elevada dependencia exterior, con un abastecimiento energético demasiado vulnerable. Este plan tiene tres objetivos básicos:

- Mayor autoabastecimiento energético.

- Impulso del ahorro y eficiencia energética.

- Utilización óptima de los recursos.

Para reducir la dependencia del petróleo y conseguir los objetivos de consumo de energía primaria, este nuevo PEN favorece las energías nacionales, caso del carbón y de la energía hidráulica que pasan de una contribución del $16,2 \%$ y $9,4 \%$, respectivamente en el PEN 1978-87, a un $25,2 \%$ y un $11,3 \%$ en el PEN 1983-92 (SOLANA, 1983; CORTINA GARCÍA, 1995).

Estos tres planes energéticos (1975-85, 1978-87 y 1983-92) abordan la planificación energética con una timorata confianza en los recursos nacionales y con poca convicción en reducir la vulnerabilidad exterior del petróleo, por la ausencia de armas eficaces que pudieran reducir tal dependencia. Bastaba con un acontecimiento político significativo en el mundo árabe, como ocurrió con el cambio de régimen político en Irán en 1979, para provocar inmediatamente una subida incontrolada del precio del crudo y la consiguiente revisión del PEN 1978. La consabida escasa contribución de los recursos energéticos autóctonos, basados casi exclusivamente en el carbón, resultaba insuficiente a todas luces. 


\section{LAS ENERGIAS RENOVABLES Y LA NECESIDAD DE PROTECCIÓN MEDIOAMBIENTAL}

Hasta muy avanzado el siglo XIX, las energías renovables podían cubrir las demandas energéticas que la sociedad requería. A raíz de la Revolución Industrial, y ante el incesante consumo energético, estas energías se vieron desplazadas por el carbón, el petróleo y el gas natural, con un mayor poder energético. Desde entonces, el consumo de energías no renovables, que ha marcado el desarrollo de la sociedad, ha derivado en el deterioro ambiental, hasta alcanzar cotas muy altas.

La energía, pues, tiene que afrontar una nueva situación que surge con la agotabilidad de las energías no renovables y la creciente degradación ambiental: el aprovechamiento de nuevos recursos energéticos renovables y una mayor atención a la protección medioambiental.

Las energías renovables se derivan, de manera directa e indirecta, de la energía que procede del sol (excepto la geotérmica y de las mareas). De este modo, el origen solar de estas energías caracteriza, por tanto, su inagotabilidad, su disponibilidad periódica y su utilización en una variada tipología de recursos energéticos, con diferente grado de aprovechamiento.

Existen un conjunto de características de las energías renovables, que van a limitar y dificultar su utilización y aprovechamiento, como son:

a) El desigual reparto por las distintas zonas del mundo.

b) Por su carácter discontinuo, la captación de energía se realiza de forma secuencial, con paradas frecuentes en la alimentación energética.

c) Son aleatorias, es decir, dependen de las condiciones meteorológicas que aún no pueden predecirse con exactitud, por lo que es difícil saber cuanto tiempo van a poder ser utilizadas (IDAE, 1992).

Por otro lado, los modelos de producción energética convencionales están centralizados en grandes unidades basadas en redes de distribución que hoy empiezan a resultar inadecuadas, por lo que se deberia pensar más bien en sistemas de distribución en los que el consumo se facilite por la proximidad de los centros de producción energética. Se trataría, pues, de diversificar los consumos procedentes de pequeñas unidades de producción, que no necesitan de gravosas inversiones, y sí de pequeñas inversiones, más factibles que los costosos procesos de producción centralizada. 
Ante este panorama las energías renovables presentan las siguientes alternativas (PIORNO/ORDAZ, 1993; PARDO ABAD, 1993; IDAE, 1988):

- El agua, el sol, el viento o la biomasa son recursos energéticos que se encuentran distribuidos por extensas áreas, lo que da lugar a una utilización descentralizada. Sin embargo, aunque se trata de energías universales, no afectan a todas las áreas del planeta por igual.

- La posibilidad de instalar pequeñas unidades energéticas de bajo coste a través de las tecnologías de conversión modulares, aunque en ningún momento se trata de competir con las grandes instalaciones energéticas.

- Los procesos de obtención o conversión energética, a través de estas fuentes, y su utilización tienen un escaso impacto ambiental, sobre todo si lo comparamos con las energías primarias convencionales, lo que permite una mejor conservación de los paisajes geográficos.

- Los procesos tecnológicos son de relativa sencillez, lo que permite un desarrollo autóctono de los mismos, asociados a beneficios industriales propios.

- Los recursos energéticos son inagotables y no plantean los problemas a largo plazo que plantean los recursos finitos. Su inagotabilidad procede de su origen solar. La cantidad de radiación solar que recibe la tierra varía con la latitud, la altura del sol y el calendario estacional.

- La energía renovable presenta un carácter intermitente. Esta utilización periódica se puede aprovechar para satisfacer las necesidades de energía final de la población.

- Son energías que no contaminan. No emiten $\mathrm{CO}_{2}$, lo que permite atenuar el efecto invernadero. En el caso de la biomasa las emisiones de $\mathrm{CO}_{2}$ proceden de la propia atmósfera, previamente absorbido a través del proceso de fotosíntesis. Por otro lado, la ausencia de contaminantes atmosféricos no favorece la lluvia ácida.

- Son especialmente competitivas para suministrar electricidad a los pequeños consumidores aislados de la red eléctrica.

El desarrollo de las energías renovables está ligado, pues, a la preocupante degradación ambiental debida a la excesiva utilización de recursos energéticos fósiles. En este sentido, la puesta en escena de las energías renovables aparece con claras ventajas como son el bajo coste ambiental y su inagotabilidad. Al mismo tiempo, el conjunto de estas ener- 
gías destaca por su carácter autóctono lo que contribuye al autoabastecimiento y a la independencia energética.

\title{
EL PEN 1991/2000: EL PLAN DE AHORRO Y EFICIENCIA ENERGÉTICA (PAEE)
}

Este nuevo plan está conformado sobre objetivos bien definidos para atender la nueva situación energética: minimización de los costes, diversificación de fuentes, potenciación de los recursos autóctonos y protección medioambiental.

EI PEN 91 está estructurado en 5 grandes campos de actuación básica: escenario internacional, demanda energética, oferta energética, energía y medio ambiente y política de I+D. Además contempla dos programas recogidos en dos anexos: el Plan de Ahorro y Eficiencia Energética y el Plan General de Residuos Radiactivos.

El Plan de Ahorro y Eficiencia Energética (PAEE) se puede definir como:

\begin{abstract}
"el conjunto de medidas tendentes a lograr una utilización más racional de la energía, bien con actuaciones sobre la demanda que aseguren un menor consumo de energía para los mismos niveles de actividad económica y de bienestar de los ciudadanos, bien con la promoción de nuevas modalidades de oferta energética (cogeneración y energías renovables) por agentes distintos a los tradicionales, con efectos beneficiosos sobre el autoabastecimiento, la eficiencia y la conservación del medio ambiente» (PEN, p. 179).
\end{abstract}

La eficiencia energética es la respuesta al ahorro y se consigue en la medida que se reduce el consumo de energía final por unidad de PIB. Por el desarrollo de la eficiencia energética está prevista una reducción del $12 \%$ en la ratio consumo de energía primaria/PIB a lo largo del periodo del PEN (PEN 1991-2000). Los Planes de Ahorro de los países comunitarios responden a:

- una escasa dotación en fuentes de energía, sobre todo en petróleo,

- una mejora de la competitividad en los distintos sectores económi$\cos , y$

- por necesidades medioambientales. 
Por tanto, conviene recordar la llamada del Consejo Mundial de la Energía en su informe sobre "La energía para el mundo de mañana", en el que establece las prioridades energéticas de los países industrializados, basadas en:

- la constante mejora de la eficiencia energética,

- el avance tecnológico,

- la progresiva reducción de los niveles de contaminación,

- la obligatoriedad de adoptar medidas cautelares sobre el cambio climático.

EI PAEE se estructura bajo dos objetivos básicos:

a) ahorro de la demanda de energía final,

b) sustitución de Producción Eléctrica Independiente (PEI).

Para el año 2000 el PAEE tiene previsto reducir en un 7,6\% la demanda de energía final, lo que supondrá 6,3 millones de Tep. Este objetivo supone la disminución de las demandas sectoriales, así como del consumo de energías fósiles (22,6\% en el carbón, $11,4 \%$ en los productos petrolíferos) y de la electricidad (5,9\%). Para cubrir la demanda de energía final tendrá lugar un crecimiento de un $26,5 \%$ del consumo de gas natural (PEN 1991-2000).

Las cuatro actuaciones del PAEE se concretan en los siguientes programas: de ahorro, de sustitución, de cogeneración y de energías renovables.

a) El programa de ahorro supone disminuir la demanda de energía final sin rebajar los niveles de actividad económica o de bienestar.

b) El programa de sustitución contempla la sustitución de productos petrolíferos y carbón, por gas natural. En este sentido, el PEN apuesta rotundamente por el sector gasista.

c) El programa de cogeneración o procesos en los que se produce y utiliza calor y electricidad conjunta y simultáneamente. Se trata de aprovechar el calor residual de la generación de electricidad.

En España la cogeneración es una práctica tradicional en industrias intensivas en energía, principalmente en los sectores químico y alimentario, sobre todo en las azucareras. El procedimiento consiste en ahorrar energía primaria en la generación de electricidad y sustitución de combustibles por el calor residual que es susceptible de aprovechamiento. En el sistema tradicional para la producción de electricidad se requiere un consumo mayor 
de energía primaria. Esta autogeneración de electricidad por el propio usuario permite un ahorro de las pérdidas por transporte y distribución.

d) El programa de energías renovables destaca por tres aspectos relevantes:

- Ventajas medioambientales por la sustitución de energías fósiles.

- Impulso de nuevas tecnologías que mejoran la comercialización de estas energías.

- Contribución al autoabastecimiento energético y a la diversificación en el aprovisionamiento de fuentes de energía.

EI PAEE contempla un aumento de las energías renovables del $20,6 \%$ entre los años 1990 y el 2000, y aportarán un total de 2,9 MTep, según previsiones del PEN. En la cobertura de demanda de energía primaria, la contribución de estas energías debería crecer, según estas previsiones, en un $45 \%$ al pasar del $2,7 \%$ en 1990 al $3,2 \%$ en el 2000 (PEN 19912000).

En España, las energías renovables, juegan un papel cada vez más importante y ocupan una destacada posición con respecto al resto de países de la Unión Europea. Se observa un creciente aumento de las investigaciones e iniciativas de proyectos orientados al desarrollo de las FER (Fuentes de Energías Renovables), con el fin de conseguir para el año 2010 que las energias renovables signifiquen el $12 \%$ del consumo energético nacional.

Según se puede observar en el siguiente cuadro, se han superado los objetivos previstos en el PAEE en cuanto a producción y potencia instalada:

PRODUCCIÓN DE ENERGÍA ELÉCTRICA CON ENERGÍAS RENOVABLES

PRODUCCIÓN (GWH/AÑO) POTENCIA (MW)

Minihidráulica

Objetivo 1991/2000

2.474

779

Realizado y en ejecución 31.12.96

$2.587,6$

732,7

$\%$ sobre objetivo

$104,6 \%$

$94,1 \%$

RSU

Objetivo 1991/2000

$1.297,5$

239

Realizado y en ejecución 31.12.96

686,5

94,1

$\%$ sobre objetivo

$52,9 \%$

$39,4 \%$ 


\section{PRODUCCIÓN DE ENERGÍA ELÉCTRICA CON ENERGÍAS RENOVABLES (Continuación)}

\begin{tabular}{lcc}
\hline & PRODUCCIÓN (GWH/AÑO) & POTENCIA (MW) \\
\hline Eólica & & \\
Objetivo 1991/2000 & 403 & 168 \\
Realizado y en ejecución 31.12.96 & $1.536,9$ & 647,5 \\
\% sobre objetivo & $381,4 \%$ & $385,4 \%$ \\
Solar fotovoltaica & & \\
Objetivo 1991/2000 & 4,5 & 2,5 \\
Realizado y en ejecución 31.12.96 & 6,7 & 3,9 \\
\% sobre objetivo & $148,9 \%$ & $156 \%$ \\
Total & & $1.188,5$ \\
Objetivo 1991/2000 & 4.179 & $1.478,2$ \\
Realizado y en ejecución 31.12.96 & $4.817,7$ & $124,4 \%$ \\
\% sobre objetivo & $115,3 \%$ & \\
\hline
\end{tabular}

Fuente: Ministerio de Industria y Energía

De este modo, en la distribución por áreas de la producción eléctrica generada con energías renovables, es de destacar como en todas ellas se han superado ampliamente, ya en 1996, los objetivos previstos en el Plan, mientras que en el caso de los RSU se observa una evolución mucho más lenta.

Para optimizar la oferta y la demanda de energía el PAEE recoge las orientaciones comunitarias sobre conservación de la energía. En este sentido, la vinculación española a programas europeos como VALOREN y THERMIE, con los que se quiere conseguir un uso más racional de la energía, ha supuesto la reducción de la dependencia energética. Las actuaciones del PAEE están orientadas a la consecución y mantenimiento de la eficiencia energética, lo que significará mejorar la competitividad empresarial y conseguir la deseada calidad medioambiental en una economía sostenible para paliar los efectos de la contaminación atmosférica, derivados de la producción y el consumo de energía, fundamentalmente por las emisiones de $\mathrm{CO}_{2}$, seguidas muy de cerca por el Panel Intergubernamental del Cambio Climático (PICC), organismo bajo los auspicios de la Organización Metereológica Mundial (OMM) y el Organismo de las Naciones Unidas para el Medio Ambiente. 


\section{LAS ENERGIAS RENOVABLES EN EL CONTEXTO COMUNITARIO}

El compromiso de desarrollar estas energias por parte de los Estados miembros de la UE, quedó de manifiesto en un documento conjunto «La Declaración de Madrid" (marzo de 1994), donde se aprobó un Plan de Acción para las Fuentes de Energías Renovables en Europa. Esta Declaración recoge las recomendaciones de la Conferencia de Naciones Unidas sobre Medio Ambiente, celebrada en Río, así como los principios de la Carta Europea de la Energía, y fija como un objetivo prioritario e inmediato:

«En el año 2010 las Fuentes de Energías Renovables pueden, y con la colaboración de todos los actores involucrados deben, sustituir el equivalente al $15 \%$ de la demanda de energía primaria convencional en la Unión Europea" (Declaración de Madrid, 1994).

Las líneas de acción de esta Declaración se concretarán en acciones políticas, legislativas y administrativas, financieras y fiscales, tecnológicas y de información, educación y formación. La contribución futura de estas energías a nivel mundial, cuyo consumo energético casi se ha triplicado en las tres últimas décadas, se considera como uno de los cuatro actores energéticos principales, tal y como expresa el Consejo Mundial de la Energía.

Las acciones tecnológicas posibilitarán tales propósitos, y los esfuerzos realizados por las Instituciones europeas y Gobiernos nacionales están dando sus frutos en programas como JOULE, THERMIE, ALTENER, VALUE, VALOREN y STOA a nivel europeo y se piensa continuarlos y ampliarlos.

El desarrollo de las Fuentes de Energia Renovables (FER) en España está ligado a la implicación en estos programas, de modo que a finales de 1995 estas FER aportaron al balance energético nacional más de seis millones de Tep/año, lo que significa aproximadamente un 6\% (IDAE, 1996). El interés suscitado por la potenciación de las FER en España resulta evidente por los más de 2.900 proyectos que vienen ejecutándose desde 1991. Se trata de un sector de gran importancia económica, social y medioambiental.

La política energética actual potencia las FER, entre otras razones, por:

- La obligada reducción de las emisiones de dióxido de carbono. Es bien sabido que estas energías provocan un menor impacto ambiental frente a las energías convencionales. 
-El desequilibrio entre áreas desarrolladas y subdesarrolladas con respecto al consumo de energía.

- La creciente crisis medioambiental. Existen elevados costes ambientales y agotamiento de los recursos por el actual modelo de consumo energético.

La investigación y desarrollo de las energías renovables, fomentada a través de las políticas energéticas, ha sido posible gracias a la creciente toma de conciencia social de conseguir una mejora en las condiciones medioambientales.

- La energía minihidráulica presenta buenas perspectivas al evitar los grandes problemas medioambientales de las grandes centrales hidráulicas y por su capacidad de aprovechamiento en pequeñas instalaciones dispersas por el territorio. Se consideran minicentrales, aquellas cuya potencia es menor a $10 \mathrm{MW}$ (R.D. 2366/1994). En los dos últimos años se han puesto en funcionamiento en España 51 minicentrales hidroeléctricas, lo que supuso un aumento de $102 \mathrm{MW}$ en la potencia instalada, gracias a las ayudas concedidas por programas comunitarios, de las que la mayor parte (en total 29) corresponden a rehabilitaciones de antiguas centrales (IDAE, 1996a).

- La energía solar térmica, en su modalidad de baja temperatura, se aprovecha mediante colectores solares que calientan agua, por lo que el sector doméstico es el principal usuario. España es uno de los países europeos que ofrecen mayores niveles de radiación solar. El programa comunitario THERMIE incluye los proyectos de electricidad solar térmica, en la modalidad de alta y media temperatura (IDAE, 1996e).

- En el seno de la UE la energía solar fotovoltaica tiene una implantación muy desigual. En España, la potencia instalada, tanto en sistemas aislados como los conectados a red, alcanzaba a finales de 1995 un total de $6,5 \mathrm{MW}$, destacando la comunidad andaluza por su potencial instalado, sobre todo en el ámbito rural. En Castilla-la Mancha, se ha instalado recientemente una planta fotovoltaica en Toledo PV, que representa el 92\% del total de la región (IDAE, 1996f).

- La biomasa es la fuente energética de mayor producción del grupo de las renovables. Cuantitativamente supone un $60 \%$ en el total de las FER, y en el balance energético global contribuye con algo más del $3 \%$. En España, la biomasa como recurso energético potencial, se evalúa en algo más de 18,4 millones de Tep/año, dividido en varios grupos: 
- biomasas agrícola y forestal,

- biomasas procedentes de las industrias forestales o agrícolas con aplicación energética,

- residuos agroganaderos, aguas residuales urbanas, es decir residuos biodegradables,

- cultivos energéticos: producción de cardos o chopos en terrenos de barbechos, cereales con producción de bioalcohol y girasol para elaborar biodiesel.

EI IDAE ha evaluado en 1994 el consumo energético de biomasa en 3,5 millones de Tep, incluyendo los sectores doméstico e industrial (IDAE, 1996c)

- En España se producen anualmente más de 14 millones de toneladas de Residuos Sólidos Urbanos, y únicamente el $4,4 \%$ se destina a la recuperación de energia, mediante el proceso de incineración. Las principales plantas están localizadas en Barcelona, Gerona, Tarragona, Madrid y Palma de Mallorca (IDAE, 1996d).

- La energía eólica, de gran crecimiento a nivel mundial, tanto por potencia instalada como por desarrollo tecnológico. En España el PAEE recoge en su programa de Energías Renovables el aprovechamiento de esta energía en zonas geográficas de alto potencial, donde están instalados parques eólicos conectados a la red eléctrica: Tarifa, Fuerteventura y Lanzarote, Cabo Villano (Galicia), Aragón, Sierra del Perdón (Navarra) y Bajo Ebro (Cataluña) (IDAE, 1996b). El auge de este sector se manifiesta en la superación con creces de los objetivos previstos en el PAEE (cp. cuadro).

Un gran inconveniente para la implantación y desarrollo de las energias renovables es el carácter especialmente disperso de las instalaciones. La innovación tecnológica permitirá su accesibilidad y hacerlas competitivas con las energias convencionales y la difusión tecnológica es un factor determinante para poder alcanzar producciones industriales de gran tirada, con el fin de conseguir un menor coste de las instalaciones y una mayor distribución espacial de éstas. El Instituto de Diversificación y Ahorro Energético (IDAE) se encarga de canalizar las inversiones en este área y realiza una difusión de sus principales ventajas y características, tanto entre los consumidores como entre los productores de energía. Al mismo tiempo se llevan a cabo estudios de los objetivos propuestos en los Planes Energéticos, a través de sus publicaciones.

En general, estas energías aún no han alcanzado un coste competitivo, y se espera en los próximos años, a pesar de su progresivo desarrollo, 
una aportación marginal en los países desarrollados. De cualquier manera, el aprovisionamiento energético de fuentes renovables, recogido en la Carta Europea de la Energia, está muy vinculado a una acción global comunitaria de protección medioambiental, que aplicada a España supone la progresiva disminución de las emisiones de $\mathrm{SO}_{2}$ en un $21 \%$ en el año 1993 , un $40 \%$ en el 98 y un $50 \%$ en el 2003 ; en las emisiones de $\mathrm{NOx}$ el descenso se establece en un $20 \%$ para 1993 y en un $40 \%$ para 1998 (PEN 1991-2000).

Para amortiguar el efecto invernadero, la Comisión de Energía Comunitaria ha aprobado estabilizar las emisiones de $\mathrm{CO}_{2}$ para el año 2000 en los niveles actuales. No podemos olvidar que, ante la incertidumbre de los riesgos, están en marcha tres planes internacionales de investigación:

- World Climate Ressearch Programme (WCRP).

- Internacional Geosphere Biosphere Programme (IGBP).

- Global Climate Observing System (GCOS).

Las exigencias medioambientales han llevado a la UE a la aprobación de programas integramente dedicados a las energías renovables, como ha sido la aprobación del Programa ALTENER en el Consejo de Ministros de las Comunidades Europeas (13 de septiembre de 1993) (IDAE, 1996). En este programa se fijan los objetivos a conseguir por la aportación de las energías renovables, lo que significaría la reducción de un total de 180 millones de toneladas de $\mathrm{CO}_{2}$ para el año 2005, y supondría en la estructura de la demanda de energía primaria, que las energías renovables pasarán de contribuir en un $4 \%$ en 1991 a un $8 \%$ en el 2005 , lo que equivaldría evitar el $10 \%$ del consumo de combustibles fósiles en términos de energía primaria.

La gravedad de los problemas ambientales impone, pues, una creciente potenciación de las energías renovables. El medio ambiente ha dejado de ser un factor limitativo del crecimiento, para convertirse en un fiel aliado del desarrollo. El objetivo no es otro que alcanzar en el campo energético una significativa aportación, aunque es necesario una confluencia de intereses de variada índole (ambientales, tecnológicos, económicos, políticos y sociales). En esta convergencia de intereses es de especial importancia la rentabilidad que cabe esperar de los aspectos ecológicos y económicos, que consideramos indispensables para el desarrollo futuro de este sector energético (Banco Mundial, 1992).

No se puede satisfacer el creciente desarrollo económico, si no se responde con proyectos energéticos encuadrados en la sostenibilidad am- 
biental. Ahora bien, las futuras expectativas de un crecimiento socialmente justo deben encauzarse en un marco cautelar que tenga capacidad de combatir tanto el desmesurado optimismo científico tecnológico, como el apasionado catastrofismo ecologista. En este sentido, la apuesta de las energías renovables es una clara invitación a valorar más racionalmente el consumo energético con criterios de ahorro y de eficiencia.

De este modo, la contribución de las energías renovables dependerá de la capacidad de innovación que posibilite aprovechar al máximo este potencial energético y que las pueda hacer competitivas frente a las energías convencionales (IDAE, 1993).

\section{BIBLIOGRAFIA UTILIZADA}

Cortina Garcia, J. (1995): La planificación energética en España. En: Economía industrial, n.ำ 302,1995 , pp. $45 / 68$.

INFORME SOBRE EL DESARROLLO MUNDIAL (1992): Desarrollo y Medio Ambiente. Banco Mundial

Instituto para la Diversificacion y AHORro de la Energía (IDAE) (1988): Guía Didáctica de la Energia. MOPU. Madrid.

INSTITUTO PARA LA DIVERSIFICACIÓN Y AHORRO DE LA ENERGIA (IDAE) (1993): Energías renovables en España. Anuario de Proyectos 1992.

InStituto PARA LA Diversificación Y AHORRo de LA ENERgia (IDAE) (1996a): Manuales de Energias renovables. Manual de Minicentrales hidroeléctricas n. 9 1. Madrid.

Instituto para la Diversificación y AHORRo de la EnERgí (IDAE) (1996b): Manuales de Energias renovables. Manual de Energía Eólica n.․ 2. Madrid.

Instituto para la Diversificacion y AhorRo de la Energia (IDAE) (1996c): Manuales de Energias renovables. Manual de Energía de la Biomasa n. 93 . Madrid.

Instituto para LA Diversificación Y AHORRO DE LA ENERGia (IDAE) (1996d): Manuales de Energías renovables. Manual de Incineración de Residuos Sólidos Urbanos n. 9 4. Madrid.

INSTITUTO PARA LA DIVERSIFICACIÓN Y AHORRO DE LA ENERGIA (IDAE) (1996e): Manuales de Energias renovables. Manual de Energía Solar Térmica.$^{\circ} 5$. Madrid.

Instituto para la Diversificación y AHORRo DE LA ENERGia (IDAE) (1996f): Manuales de Energías renovables. Manual de Energía Solar Fotovoltaica n. 96 . Madrid.

Iranzo Martín, J.E. (1992): El sector energético español. En: Papeles de Economía Española, ก.․ 50, pp. $173-180$.

Iranzo Martin, J.E. (1984): El sector energético español: realidades y posibilidades. En: Papeles de Economia Española, pp. 271-290.

PARDO ABAD, C. (1993): Las fuentes de energía. Madrid.

Pionno Hernandez, A.JF. Ordaz Oviedo (1993): Energías renovables. Aproximación a su estudio. Salamanca.

Plan Energético Nacional 1991-2000: Ministerio de Industria, Comercio y Turismo. Secretaría de la Energía y Recursos Minerales.

Plan de acción para las fuentes de Energias Renovables en Europa (1994): Declaración de Madrid. Madrid 16-17-18, marzo, 1994.

REDCLIKT, M.R. (1996): Compromisos sociales y medios ambiente. En: Información Comercial Española, núm. 751, pp. 119-227.

SOLANA, M. (1983): Alternativa socialista al PEN (1983). En: Papeles de Economía Española, núm. 14, pp. 14-20. 